\title{
The Novel GTPase Rit Differentially Regulates Axonal and Dendritic Growth
}

\author{
Pamela J. Lein, ${ }^{1,2}$ Xin Guo, ${ }^{2}$ Geng-Xian Shi, ${ }^{3}$ Melissa Moholt-Siebert, ${ }^{1}$ Donald Bruun, ${ }^{1}$ and Douglas A. Andres ${ }^{3}$ \\ ${ }^{1}$ Center for Research on Occupational and Environmental Toxicology, Oregon Health \& Science University, Portland, Oregon 97239, ${ }^{2}$ Department of \\ Environmental Health Sciences, Johns Hopkins University Bloomberg School of Public Health, Baltimore, Maryland 21205, and ${ }^{3}$ Department of Molecular \\ and Cellular Biochemistry, University of Kentucky College of Medicine, Lexington, Kentucky 40536
}

The Rit GTPase is widely expressed in developing and adult nervous systems, and our previous data with pheochromocytoma cells implicate Rit signaling in NGF-induced neurite outgrowth. In this study, we investigated a role for Rit in neuronal morphogenesis. Expression of a dominant-negative ( $\mathrm{dn}$ ) Rit mutant in hippocampal neurons inhibited axonal growth but potentiated dendritic growth. Conversely, a constitutively active (ca) Rit mutant promoted axonal growth but inhibited dendritic growth. Dendritogenesis is regulated differently in sympathetic neurons versus hippocampal neurons in that sympathetic neurons require NGF and bone morphogenetic proteins (BMPs) to trigger dendritic growth. Despite these differences, dnRit potentiated and caRit blocked BMP7-induced dendritic growth in sympathetic neurons. Biochemical studies indicated that BMP7 treatments that caused dendritic growth also decreased Rit GTP loading. Additional studies demonstrate that caRit increased extracellular signal-regulated kinase 1/2 (ERK1/2) phosphorylation and pharmacological inhibition of MEK1 (mitogen-activated protein kinase/ERK 1) blocked the axon-promoting and dendrite-inhibiting effects of caRit. These observations suggest that Rit is a convergence point for multiple signaling pathways and it functions to promote axonal growth but inhibit dendritic growth via activation of ERK1/2. Modulation of the activational status of Rit may therefore represent a generalized mechanism across divergent neuronal cell types for regulating axonal versus dendritic growth modes.

Key words: Rit, GTPase, axon, dendrite, ERK, sympathetic neuron, hippocampal neuron, BMP, NGF

\section{Introduction}

In vivo, the dendritic arbor often undergoes rapid expansion only after significant axonal growth has occurred, and dendrites typically project along different pathways than axons (DeFelipe and Jones, 1988). This spatiotemporal separation of axonal and dendritic growth is thought to reflect spatiotemporal differences in the expression of extrinsic axon- and dendrite-specific cues (Prochiantz, 1995; Higgins et al., 1997; McAllister, 2000; Scott and Luo, 2001). However, reports that retinal ganglion cells switch their responsiveness to neurotrophins from axonal to dendritic growth during development (Goldberg et al., 2002) suggest an alternative model in which extrinsic signals that support axonal and dendritic growth are generally available in the environment, and the morphogenic response of the neuron to these cues is determined by its intrinsic state (Goldberg, 2004). Observations that activity-induced axonal versus dendritic growth is mediated by different CaM-kinase isoforms (Redmond et al., 2002; Fink et al., 2003; Wayman et al., 2004) suggest that modulation of

\footnotetext{
Received Sept. 8, 2006; revised March 19, 2007; accepted March 20, 2007.

This work was supported by National Institutes of Health (NIH)-National Institute of Neurological Disorders and Stroke Grants NS046649 (P.J.L.) and NS045103 (D.A.A.), by the COBRE program of the National Center for Research Resources (Grant P2ORR20171 to D.A.A.), and by NIH-National Institute of Environmental Health Sciences Training Grant ES07141 (X.G.).

Correspondence should be addressed to Dr. Pamela Lein, Center for Research on 0ccupational and Environmental Toxicology/L606, Oregon Health \& Science University, 3181 Southwest Sam Jackson Park Road, Portland, OR 97239. E-mail: leinp@ohsu.edu.

DOI:10.1523/JNEUROSCI.5633-06.2007

Copyright $\odot 2007$ Society for Neuroscience $\quad$ 0270-6474/07/274725-12\$15.00/0
}

intracellular signaling cascades may represent a mechanism for regulating axon- versus dendrite-specific interpretations of extrinsic cues (Goldberg, 2004), but this mechanism has not been widely tested beyond this example.

Rit belongs to a recently described subgroup of the Ras subfamily of GTPases (Reuther and Der, 2000). Originally cloned from mouse (Lee et al., 1996) and human (Shao et al., 1999) retina, Rit transcripts have subsequently been detected in embryonic, postnatal, and adult murine brain (Lee et al., 1996), in diverse regions of the human fetal brain (Wes et al., 1996), and in primary cultures of sympathetic neurons (Spencer et al., 2002b). Despite its widespread occurrence in the nervous system, its cellular function(s) in neurons is not known. Ras GTPases respond to external signals by exchanging GTP for bound GDP, which enables interaction with downstream effector proteins, thereby linking extracellular signals to specific intracellular signaling cascades that mediate diverse cellular functions, including cell differentiation (Reuther and Der, 2000). We recently demonstrated that nerve growth factor (NGF) activates Rit and that Rit-GTP contributes to NGF-induced neurite growth in pheochromocytoma and SH-SY5Y cells (Spencer et al., 2002b; Hynds et al., 2003; Shi and Andres, 2005), suggesting that Rit signaling may be involved in controlling neuronal morphogenesis. The finding that Rit associates with the Par3-Par6 complex (Hoshino et al., 2005), which is involved in axon-dendrite specification in cultured hippocampal neurons (Shi et al., 2003), also supports a role for Rit in neuronal morphogenesis. In this study, we demonstrate that 
modulating Rit signaling using molecular or pharmacological approaches differentially alters axonal and dendritic growth. We identify Rit as a novel downstream target of bone morphogenetic protein 7 (BMP7), an extrinsic cue required for dendritic growth in sympathetic neurons (Lein et al., 1995) that also enhances dendritic growth in hippocampal neurons (Withers et al., 2000), and demonstrate that the effect of BMP on Rit activation is modified by NGF signaling. These data not only suggest Rit is a convergence point for multiple signaling pathways involved in controlling neuronal morphogenesis but also identify a new and unexpected role for Rit in determining axonal versus dendritic growth responses.

\section{Materials and Methods}

Materials. The construction and characterization of mammalian expression vectors that encode constitutively active Rit ${ }^{\mathrm{Q79L}}$ (caRit) or dominant-negative $\mathrm{Rit}^{\mathrm{S} 35 \mathrm{~N}}$ (dnRit) tagged with green fluorescent protein (GFP) or wild-type Rit tagged with the Flag epitope (3XFlag-RitWT) and of recombinant adenovirus vectors that express GFP alone or coexpress GFP and either caRit or dnRit have been described previously (Shao and Andres, 2000, 2005; Spencer et al., 2002b). Recombinant human BMP7 was generously provided by Curis (Cambridge, MA). 2' Amino-3'-methoxyflavone (PD98059) was purchased from Biomol (Plymouth, MA), and 1,4-diamino-2,3-dicyano-1,4-bis(o-aminophenylmercapto)butadiene ethanolate (U0126) was purchased from Promega (Madison, WI). MEK [mitogen-activated protein kinase (MAP)/extracellular signal-regulated kinase (ERK) kinase] inhibitors were prepared as $1000 \times$ stocks in DMSO and diluted directly into tissue culture medium to yield final concentrations of $10 \mu \mathrm{M}$ (Kim et al., 2004). Previous studies have demonstrated that DMSO at 1:1000 does not alter hippocampal (Howard et al., 2003) or sympathetic (Howard et al., 2005) morphogenesis and does not inhibit caRit effects on neurite outgrowth of PC6 (Spencer et al., 2002a) or SH-SY5Y (Hynds et al., 2003) cells.

Cell culture and expression of cDNA. Hippocampal neurons were dissociated from the hippocampi of embryonic day 18 Holtzman rats using previously described methods (Howard et al., 2003). Briefly, hippocampi were dissociated by treatment with trypsin $(2 \mathrm{mg} / \mathrm{ml}$; Sigma, St. Louis, MO) and deoxyribonuclease $(0.6 \mathrm{mg} / \mathrm{ml}$; Sigma) for $2 \mathrm{~min}$ and treated with soybean trypsin inhibitor $(1 \mathrm{mg} / \mathrm{ml}$; Sigma) for $10 \mathrm{~min}$. Dissociated cells were plated onto glass coverslips precoated with poly-D-lysine (100 $\mu \mathrm{g} / \mathrm{ml}$; Sigma) and laminin (6 $\mu \mathrm{g} / \mathrm{ml}$; Invitrogen, San Diego, CA) and maintained in serum-free Neurobasal medium with B27 supplements (Invitrogen). Sympathetic neurons were dissociated from the superior cervical ganglia of perinatal Holtzman rats (Harlan Sprague Dawley, Rockford, IL) as described previously (Higgins et al., 1991). Cells were plated onto poly-D-lysine (100 $\mu \mathrm{g} / \mathrm{ml}$; Sigma)-coated glass coverslips and maintained in a serum-free medium containing $\beta$-NGF (100 ng/ml; Harlan Bioproducts, Indianapolis, IN).

To study effects of Rit on neuronal morphogenesis, hippocampal or sympathetic neuronal cultures were infected with adenoviral vectors expressing GFP alone or coexpressing GFP and either caRit or dnRit. Alternatively, cultures were transfected with plasmids encoding enhanced GFP, GFP-tagged caRit, or GFP-tagged dnRit using Lipofectamine 2000 (Invitrogen) according to the manufacturer's instructions, as described previously (Kim et al., 2002). In neuronal cultures used to examine axonal growth, cultures were infected or transfected $16 \mathrm{~h}$ after plating. Infecting or transfecting cultures at significantly earlier time points (6-10 h after plating) yielded inconsistent results and often caused decreased cell adhesion or cell viability. In neuronal cultures used to study the role of Rit in dendritic growth, cytosine- $\beta$-D-arabinoside $(1 \mu \mathrm{M})$ was added to the medium of all cultures for $48 \mathrm{~h}$ beginning on day 2 to eliminate non-neuronal cells. Cultures were then infected or transfected with Rit constructs at varying times after antimitotic treatment as stated in the text. Infection efficiency ranged from 20 to 50\%; transfection efficiency typically ranged from 1 to $15 \%$ (Kim et al., 2002), depending on the construct and neuronal cell type. Comparable morphometric results were obtained using either infection with adenoviral vectors or transfection with mammalian expression vectors, but because signifi- cantly increased numbers of neurons expressing Rit constructs were obtained using adenoviral vectors, this method was used to generate the data presented in Results. Dendritic growth was induced in sympathetic cultures by adding suboptimal $(10 \mathrm{ng} / \mathrm{ml})$ or maximally effective $(50$ $\mathrm{ng} / \mathrm{ml}$ ) concentrations of BMP7 to the culture medium (Lein et al., 1995) within $24 \mathrm{~h}$ after exposure to expression vectors. To determine the role of ERK1/2 in Rit effects on neuronal morphogenesis, cultures were treated with MEK inhibitor PD98059.

PC6, a PC12 cell line that produces neurites in response to NGF but grows as isolated cells rather than in clumps, was a generous gift from Dr. T. Vanaman (University of Kentucky, Lexington, KY). The cells were maintained in DMEM (Invitrogen) containing $10 \%(\mathrm{v} / \mathrm{v})$ heatinactivated FBS (HyClone, Salt Lake City, UT), 5\% (v/v) heat-inactivated horse donor serum (Invitrogen), $100 \mu \mathrm{g} / \mathrm{ml}$ streptomycin, and $100 \mathrm{U} / \mathrm{ml}$ penicillin at $37^{\circ} \mathrm{C}$ in a humidified atmosphere of $5 \% \mathrm{CO}_{2}$. PC6 cells were transfected with Effectene (Qiagen, Chatsworth, CA) as described previously (Shi and Andres, 2005).

Immunocytochemical analyses of neuronal Rit expression. Cultures were fixed in $4 \%$ paraformaldehyde prepared in PHEM buffer (60 mM PIPES, $25 \mathrm{~mm}$ HEPES, 5 mм EGTA, $1 \mathrm{~mm} \mathrm{MgCl}_{2}$, and $3 \%$ sucrose, $\mathrm{pH} 7.4$ ), blocked in 5\% bovine serum albumin in PBS (0.1 M phosphate, $\mathrm{pH} 7.4$, and $150 \mathrm{~mm} \mathrm{NaCl}$ ), reacted with monoclonal antibody (mAb) generated against Rit (mAb R0003U; Gamma-1, Lexington, KY), and visualized using rhodamine-conjugated secondary antisera (Roche Products, Indianapolis, IN). The specificity of this Rit mAb was demonstrated in previous studies (Spencer et al., 2002b) and confirmed in these studies by establishing that precipitating the Rit $\mathrm{mAb}$ with recombinant Rit protein before reaction with either hippocampal or sympathetic neuronal cultures significantly decreased fluorescence intensity to background levels in both neuronal culture systems.

Morphological analyses. Neuronal morphology was quantified in digitized images of neurons expressing GFP by individuals blind to the experimental condition. Hippocampal neurons in culture are considered to be polarized when one of the rudimentary neurites exceeds the others by a critical length (at least 10-20 $\mu \mathrm{m}$ ) and begins to elongate rapidly (Goslin and Banker, 1989). To determine the percentage of polarized neurons in hippocampal cultures $48 \mathrm{~h}$ after plating, we used a conservative definition of polarization: a neuron was considered to be polarized if its longest neurite was at least $50 \mu \mathrm{m}$ longer than any of its other processes (Lein et al., 1992). Immunocytochemistry was used to distinguish axons from dendrites (Lein et al., 1995). Cultures were fixed with $4 \%$ paraformaldehyde, permeabilized with $0.1 \%$ Triton-X-100 in PBS, and reacted with polyclonal antibodies that react with GFP (Invitrogen, Eugene, OR) and mAbs that react with either axon- or dendrite-selective antigens. To identify dendrites, both cultured hippocampal and sympathetic neurons were immunostained with SMI-52, a mAb specific for MAP2 (Sternberger Immunocytochemicals, Baltimore, MD). Axons in hippocampal neurons were identified using a mAb to tau-1 (Chemicon, Temecula, $\mathrm{CA})$, and axons in sympathetic neurons were identified using a mAb to phosphorylated forms of the heavy (NF-H) and medium (NF-M) neurofilament subunits (SMI-31; Sternberger Immunocytochemicals). Axonal and dendritic growth was quantified using SPOT imaging software (Diagnostic Instruments, Sterling Heights, MI).

Cell viability assay. Varying times after infection with adenoviral vectors, neuronal cell cultures were incubated with propidium iodide (6.3 $\mathrm{ng} / \mu \mathrm{l}$; Sigma) in $\mathrm{L} 15$ medium for $15 \mathrm{~min}$ at $37^{\circ} \mathrm{C}$. Nonviable cells take up the membrane-impermeant dye propidium iodide, which binds nucleic acids to yield nuclear red fluorescence (Vaughan et al., 1995). The number of viable and nonviable GFP+ cells was visualized in 10 randomly chosen fields (at $100 \times$ ) per experimental condition using a Nikon (Tokyo, Japan) Eclipse E400 microscope equipped with epifluorescence, and digital images captured with a Nikon DXM1200 digital camera were quantified using MetaMorph imaging software (Universal Imaging, Downingtown, PA). The percentage of viable cells per field was calculated as (total number of GFP + cells evaluated per field) - (number of GFP + cells with nuclear red fluorescence per field)/(total number of GFP + cells evaluated per field) $\times 100$. Data are expressed as the mean \pm SEM.

Western blot analyses. Cultured hippocampal neurons were infected 
with Ad-GFP or Ad-caRit for $16 \mathrm{~h}$. PD98059 (10 $\mu \mathrm{M})$ was added to a subset of cultures within $6 \mathrm{~h}$ after infection, and $48 \mathrm{~h}$ later, cells were lysed in buffer A (20 mM Tris, pH 7.6, $250 \mathrm{~mm} \mathrm{NaCl}, 2.5 \mathrm{~mm}$ EDTA, $3 \mathrm{~mm}$ EGTA, $20 \mathrm{~mm}, \beta$-glycerol phosphate, $1 \mathrm{~mm}$ vanadate, $50 \mathrm{~mm} \mathrm{KF}$, and $1 \times$ protease inhibitor mixture from Calbiochem, La Jolla, CA). Lysates were centrifuged at $15,000 \times g$ for $10 \mathrm{~min}$, and the protein concentration of the supernatant was determined using the Bradford assay (Bio-Rad, Hercules, CA). Equal amounts of protein from each lysate were separated by SDS-PAGE (10\%), transferred to nitrocellulose membranes, and probed with antibodies specific for ERK1/2 or phosphorylated ERK1/2 (Cell Signaling Technology, Beverly, MA). Antigen-antibody complexes were detected using horseradish peroxidase-conjugated secondary antibodies (Boehringer Mannheim, Indianapolis, IN) and an enhanced chemiluminescent reagent (Amersham Biosciences, Piscataway, NJ).

Rit-GTP precipitation assays. Glutathione $S$-transferase (GST) fusion proteins containing the Rit binding domain of RGL3 (residues 610-709) were expressed and purified, and Rit activation was assessed in PC6 cells or primary hippocampal cultures as described previously (Shi and Andres, 2005). PC6 cells seeded in six-well plates were transfected with $1 \mu \mathrm{g}$ of $3 x$ Flag-Rit-WT and incubated for an additional $36 \mathrm{~h}$ to allow maximal gene expression. Cells were then grown in serum-free DMEM for an additional $5 \mathrm{~h}$. Hippocampal neurons were transfected with 3xFlagRit-WT at the time of plating using the Amaxa Biosystems (Gaithersburg, MD) nucleofection system as directed by the manufacturer. Cultures were maintained in Neurobasal medium supplemented with B27 for $72 \mathrm{~h}$ before treatment. Cell monolayers were washed once in ice-cold PBS and lysed in GST pull-down assay buffer (20 mu HEPES, pH 7.4, 250 $\mathrm{mm} \mathrm{NaCl}, 50 \mathrm{~mm} \mathrm{KF}, 50 \mathrm{~mm} \beta$-glycerolphosphate, $1 \%$ Triton X-100, $10 \%$ glycerol, and $1 \times$ protease mixture) with sonication on ice. GST resin $(10 \mu \mathrm{g}$ of the appropriate fusion protein $/ 20 \mu$ l glutathione beads) was added to Rit-expressing cell lysates ( $200 \mu \mathrm{g}$ of total protein) in a total volume of $1 \mathrm{ml}$ and incubated with rotation for $1 \mathrm{~h}$ at $4^{\circ} \mathrm{C}$, and the resin was recovered by centrifugation at $4^{\circ} \mathrm{C}(5 \mathrm{~min}$ at $10,000 \mathrm{rpm})$. The GSTRGL3-RBD pellets were washed once with GST pull-down buffer, twice with GST pull-down buffer supplemented with $500 \mathrm{~mm} \mathrm{NaCl}$, and, finally, with two additional washes with GST pull-down buffer. Bound GTP-Rit was detected by immunoblot analysis using anti-Flag $\mathrm{mAb}$. To determine the effect of BMP7 and NGF on Rit activation, cultures expressing 3xFlag-Rit-WT were stimulated with recombinant human BMP7 (30 ng/ml) or $\beta$-NGF (100 ng/ml) (R \& D Systems, Minneapolis, $\mathrm{MN}$ ) for $10 \mathrm{~min}$. A subset of these cultures was pretreated with rat $\beta$-NGF (100 ng) or BMP7 (30 ng/ml) for $10 \mathrm{~min}, 4 \mathrm{~h}$, or $24 \mathrm{~h}$ before stimulation with BMP7.

Statistical analyses. Within each experiment, 30-50 neurons from two to three cultures were analyzed per experimental condition. Experiments were repeated in cultures from at least three separate dissections. Data are presented as the mean \pm SEM. Statistical significance was assessed by one-way ANOVA, followed by Tukey's post hoc test. ANOVA $p$ values are reported in the figure legends.

\section{Results \\ Rit is localized to axons and dendrites in hippocampal neurons}

It has been reported that Rit transcripts are present in human hippocampus (Wes et al., 1996). However, the cellular and subcellular distribution of Rit protein in the hippocampus is not known. To address this question, we used a mAb previously demonstrated to specifically recognize Rit (Spencer et al., 2002b) to localize endogenous Rit in cultured hippocampal neurons at varying stages of morphological differentiation. Hippocampal neurons derived from embryonic rat brain develop a polarized form in culture, extending a single axon and multiple dendrites even when grown in the absence of contact with target cells, glial cells, or afferent input (Bartlett and Banker, 1984). The development of this characteristic morphology follows a well defined sequence of events in culture (Dotti and Banker, 1987). During the first $24 \mathrm{~h}$ after plating, hippocampal neurons typically extend four to five short processes of approximately equal length that do not exhibit clear axonal or dendritic traits (Goslin et al., 1998). Within 24-48 h, one of these minor processes begins to grow rapidly, becoming significantly longer than the others. This process differentiates into the axon of the neuron, and at this stage, the neuron becomes polarized. Over the next several days, the remaining minor processes begin to elongate, ultimately becoming dendrites. In hippocampal neurons reacted with Rit mAb $24 \mathrm{~h}$ after plating (Fig. $1 \mathrm{~A}$ ), staining was present throughout the neuronal cell body and minor processes. Within the neuronal cell body, we typically observed an aggregate of intense Rit immunofluorescence that did not colocalize with 4',6-diamidino-2phenylindole, indicating that it was extranuclear. Within the minor processes, the growth cones were much more intensely stained than the shaft. In polarized neurons at $48 \mathrm{~h}$, Rit immunoreactivity was distributed diffusely throughout the cytoplasm of the cell body, the axon, and the minor processes with no apparent differences in staining intensity between the axon and the minor processes (data not shown). In hippocampal neurons with mature dendritic processes, Rit immunoreactivity was evident throughout the cell body, axonal plexus, and dendritic arbor (Fig. $1 B-D)$.

\section{Rit activation promotes axonal growth in hippocampal neurons via ERK1/2 signaling}

To determine whether Rit influences early morphogenic events in cultured hippocampal neurons, we quantified the effect of decreasing or increasing Rit activity on the development of neuronal polarity and on axonal growth. Rit activity was modulated in hippocampal neurons by infecting cultures $16 \mathrm{~h}$ after plating with adenovirus encoding GFP and either the dnRit ${ }^{\mathrm{S} 35 \mathrm{~N}}$ mutant or the caRit $^{\text {Q79L }}$ mutant (Spencer et al., 2002b; Shi and Andres, 2005). At $48 \mathrm{~h}$ after plating (corresponding to $24 \mathrm{~h}$ after infection), we determined the percentage of polarized GFP-positive neurons, defined as the percentage of neurons with one long process that was at least $50 \mu \mathrm{m}$ longer than any of its other processes (Lein et al., 1992). In cultures infected with control vector encoding only GFP, $98 \%$ of the GFP-positive hippocampal neurons were polarized. Among the population of uninfected neurons in the same cultures, $96 \%$ of the neurons were polarized. These findings are consistent with previous observations of hippocampal neurons cultured under comparable culture conditions in the absence of adenovirus (Lein et al., 1992). Expression of dnRit decreased the percentage of polarized neurons by $27 \%$ (Fig. $2 A$ ). In those hippocampal neurons that had become polarized, dnRit expression did not alter the number of axons extended (Fig. $2 B$ ) but did significantly decrease total axonal length to approximately half that observed in control neurons expressing GFP only (Fig. 2C). Infection with Ad-dnRit had no effect on the number or length of minor processes in polarized neurons relative to neurons infected with Ad-GFP (data not shown). Increasing Rit activity by expressing caRit in hippocampal neurons did not influence neuronal polarization at $48 \mathrm{~h}$ (Fig. $2 \mathrm{~A}$ ), nor did it alter the number of axons extended (Fig. $2 B$ ). However, caRit expression did significantly increase total axonal length by approximately twofold relative to control hippocampal neurons expressing GFP only (Fig. 2C). In PC6 cells, Rit influences cell survival (Spencer et al., 2002b), raising the possibility that the effects of dnRit or caRit on axonal growth are secondary to effects on neuronal survival. To address this possibility, cell viability, as determined by the uptake of propidium iodide (Vaughan et al., 1995), was assessed in hippocampal neurons infected with Ad-GFP, Ad-dnRit, or Ad-caRit. At $24 \mathrm{~h}$ after 

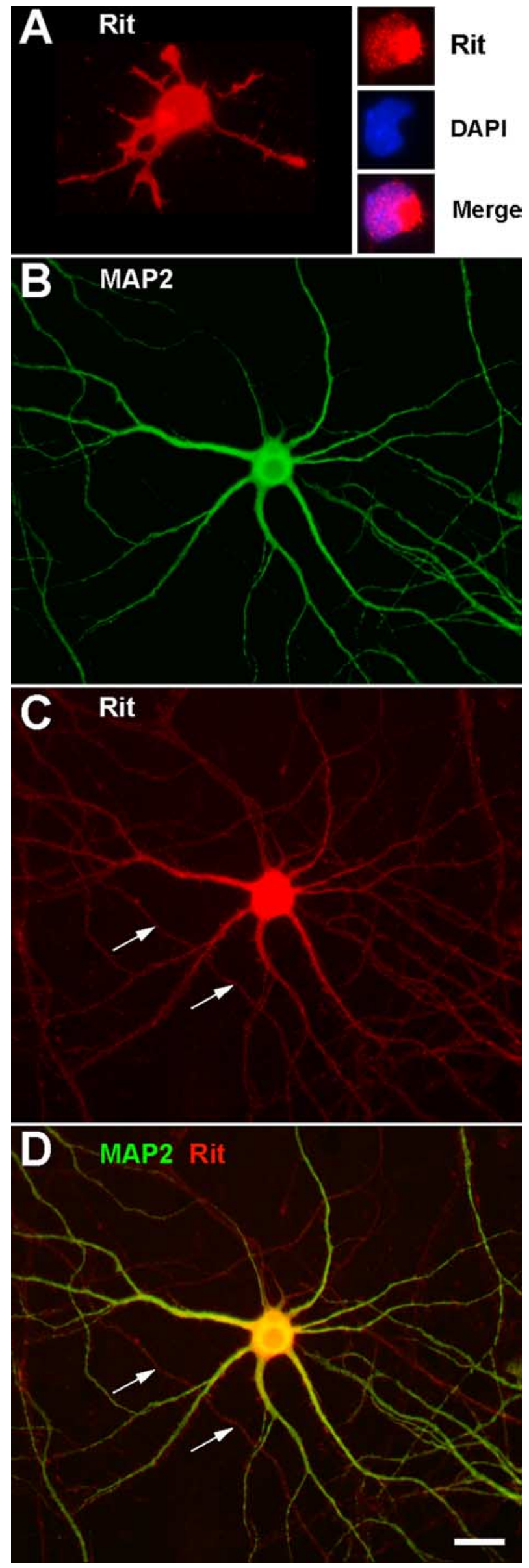

infection, neither dnRit nor caRit expression had any effect on the percentage of neuronal cell viability relative to control neurons expressing only GFP (Table 1).

We have shown previously that Rit increases neurite outgrowth in PC6 and SH-SY5Y cells via activation of MEK1, resulting in phosphorylation of ERK1/2 (Spencer et al., 2002b; Hynds et al., 2003; Shi and Andres, 2005). To determine whether caRit similarly enhances axonal growth in hippocampal neurons via activation of ERK1/2, cultures infected with Ad-GFP, Ad-dnRit, or Ad-caRit were exposed to PD98059 (10 $\mu \mathrm{M})$, a specific inhibitor of MEK1. PD98059 had no effect on axonal growth in hippocampal neurons infected with Ad-GFP or Ad-dnRit; however, PD98059 completely blocked the axonal growth induced by caRit (Fig. $2 B, C$ ). Similarly, the structurally unrelated MEK inhibitor U0126 (10 $\mu \mathrm{M})$ blocked caRit-induced axonal growth, resulting in axonal morphology comparable to control neurons expressing GFP only. To confirm that caRit activates MEK1 in neurons, lysates from hippocampal cultures infected with Ad-caRit were immunoblotted with antibodies that specifically recognize the phosphorylated form of ERK1/2. Phosphorylated ERK1/2 was observed in hippocampal cultures infected with Ad-GFP, and this was significantly reduced by PD98059 (Fig. $2 D, E$ ). Infection with Ad-caRit significantly increased levels of phosphorylated ERK1/2 relative to cultures infected with Ad-GFP, and this increase was completely blocked by PD98059 (Fig. 2D,E).

\section{Rit activation inhibits dendritic growth in hippocampal neurons via ERK signaling}

In short-term cultures of hippocampal neurons $(<48 \mathrm{~h})$, altering Rit activity influenced the growth of axons but not the growth of minor processes, suggesting that Rit selectively influences axonal morphogenesis. However, previous developmental studies of hippocampal neurons in culture have indicated that significant differentiation and growth of dendrites often does not begin until the third or fourth day in vitro (Dotti and Banker, 1987; Goslin and Banker, 1989). Thus, to determine whether Rit modulates not only early stages of morphological differentiation typified by axon growth but also later stages of neuronal morphogenesis characterized by dendritic growth, hippocampal neurons were infected with Ad-GFP, Ad-dnRit, or Ad-caRit $24 \mathrm{~h}$ after plating, and dendritic morphology was assessed $4-5 \mathrm{~d}$ after plating.

Infection of hippocampal cultures with adenoviral vectors had no effect on cell viability $5 \mathrm{~d}$ after infection (Table 1 ). Hippocampal neurons infected with Ad-GFP (Fig. $3 A, B$ ) typically expressed two to three dendrites. Expression of dnRit (Fig. 3C,D) did not alter the number of dendrites extended by hippocampal neurons (Fig. 3G); however, it did significantly increase total dendritic length by $\sim 1.4$-fold (Fig. $3 H$ ). Conversely, expression of caRit (Fig. $3 E, F$ ) significantly decreased dendritic growth in hip-

\section{$\leftarrow$}

Figure 1. Subcellular localization of endogenous Rit in cultured hippocampal neurons. Fluorescence micrographs of hippocampal neurons immunostained for Rit (red) or MAP2 (green) are shown. $A$, After $24 \mathrm{~h}$ in culture, hippocampal neurons grown on poly---lysine and laminin typically extend multiple processes of relatively equal length. During these early stages of morphological differentiation, Rit immunoreactivity is evident not only in the cytoplasm of the neuronal somata but also in all of the minor processes. At these early stages of growth, the most intense Rit immunoreactivity is associated with the growth cones and with an aggregate localized to the somata. Double staining for Rit and DAPI (4',6-diamidino-2-phenylindole; inset) indicates that this somatic Rit aggregate is extranuclear. $\boldsymbol{B}-\boldsymbol{D}$, In more mature hippocampal neurons ( $12 \mathrm{~d}$ in vitro) that express fully differentiated axons and dendrites, diffuse Rit immunoreactivity is evident in the cell body, throughout the extensive dendritic arbor (identified as MAP2-immunoreactive processes) and within the axonal shaft (arrows). Scale bar, $20 \mu \mathrm{m}$. 

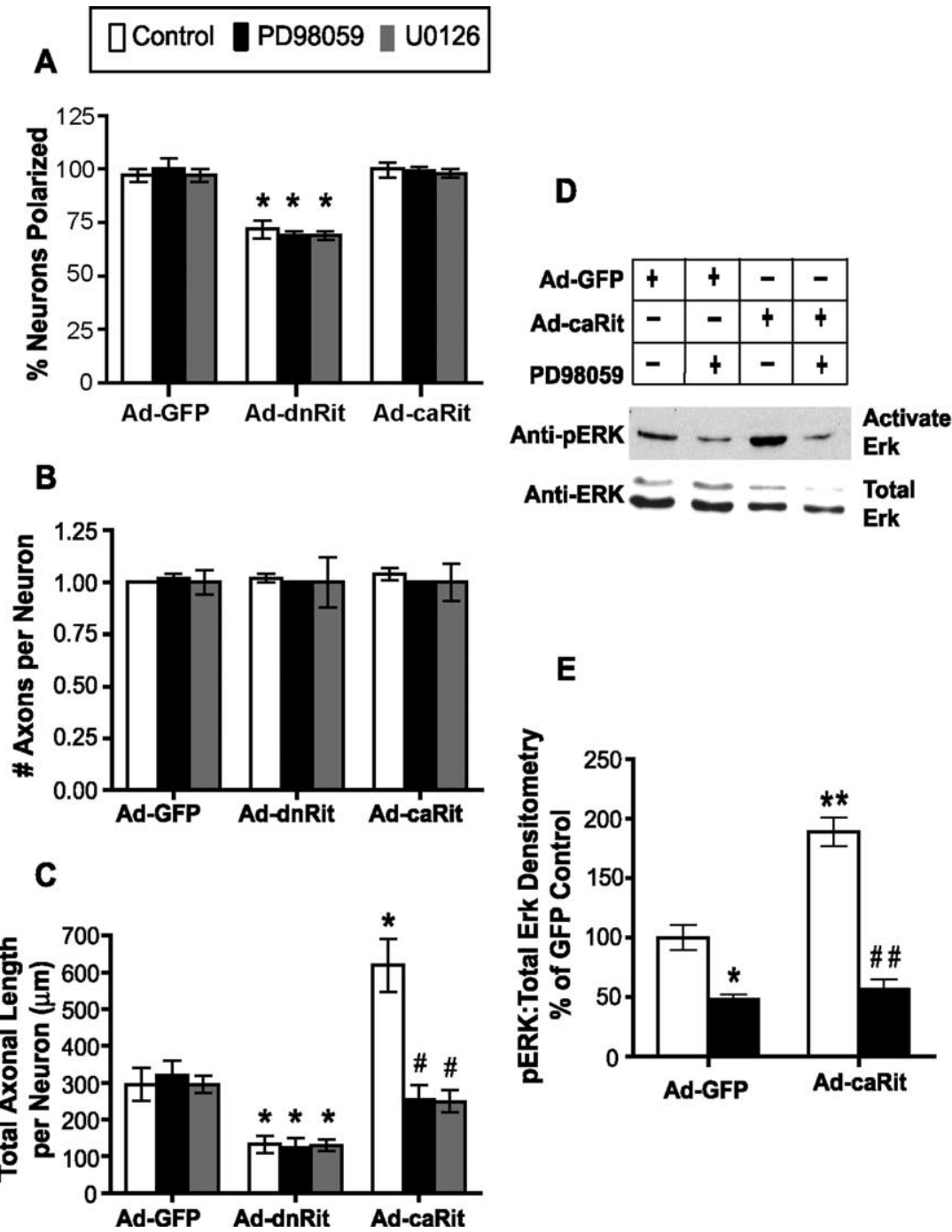

Figure 2. Activation of Rit promotes axonal growth in cultured hippocampal neurons via ERK activation. Hippocampal neurons were infected $16 \mathrm{~h}$ after plating with adenovirus expressing GFP alone (Ad-GFP) or coexpressing GFP and either dominantnegative Rit ${ }^{535 \mathrm{~N}}$ (Ad-dnRit) or constitutively active Rit ${ }^{079 \mathrm{~L}}$ (Ad-caRit) in the absence or presence of the MEK inhibitors PD98059

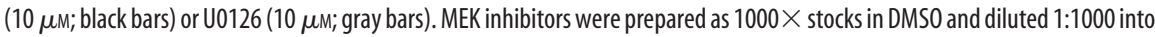
tissue culture medium. Neuronal polarity and axonal morphology were analyzed in cultures fixed and immunostained for both GFP and tau, an axonal cytoskeletal protein, $24 \mathrm{~h}$ after infection. Neurons were scored as polarized if the longest neurite was at least 50 $\mu \mathrm{m}$ longer than any of the other processes extended by the same cell. $A$, At $48 \mathrm{~h}$ after plating, expression of caRit had no effect on the percentage of GFP + neurons that were polarized, but expression of dnRit significantly inhibited the percentage of polarized GFP + neurons. * $p<0.05$ versus GFP control (post hoc analysis). $\boldsymbol{B}$, Quantification of axonal growth in polarized neurons indicated that relative to control neurons expressing only GFP, expression of either dnRit or caRit had no effect on the number of axons per neuron. C, However, dnRit significantly decreased axonal length, whereas caRit significantly increased axonal length. Both PD98059 and U0126 blocked the axon-promoting effects of caRit. Independent studies confirmed that DMS0 at 1:1000 did not alter axonal length in cultured hippocampal neurons infected with Ad-dnRit (47 $\pm 4 \%$ of Ad-GFP control) or Ad-caRit ( $230 \pm 18 \%$ of AD-GFP control) relative to hippocampal cultures infected with these constructs in the absence of DMSO (48 \pm 3 and $215 \pm$ $20 \%$ of Ad-GFP controls, respectively). Data are expressed as the mean $\pm \mathrm{SEM}$ ( $n \geq 30$ neurons per experimental group). $p<0.01$ (ANOVA); ${ }^{*} p<0.05$ versus GFP control ( post hoc analysis); ${ }^{\#} p<0.05$ versus Ad-caRit control ( post hoc analysis). D, E, Representative Western blot $(\boldsymbol{D})$ and corresponding densitometric analysis $(\boldsymbol{E})$ of activated (phosphorylated) ERK (pERK) and total (phosphorylated and nonphosphorylated) ERK in lysates from hippocampal neurons infected with Ad-GFP or Ad-caRit. Infection with caRit significantly increases pERK in hippocampal cultures relative to control cultures infected with Ad-GFP. Treatment of caRit-infected cells with the MEK inhibitor PD98059 (10 $\mu \mathrm{m}$ ) inhibits caRit-induced ERK phosphorylation. Data are expressed as the mean $\pm \operatorname{SEM}$ ( $n=3$ blots obtained in 3 separate experiments performed using cultures from 3 separate dissections). $p<0.001$ (ANOVA); ${ }^{*} p<0.05$ and ${ }^{* *} p<0.01$ versus GFP control (post hoc analysis); ${ }^{* \#} p<0.01$ versus Ad-caRit control (post hoc analysis).

pocampal neurons as evidenced by an approximate twofold reduction in the number of dendrites per neuron (Fig. $3 G$ ) and an approximate 2.5 -fold reduction in total dendritic length per neuron (Fig. 3H). Inhibition of MEK by PD98059 did not change dendritic morphology in neurons infected with Ad-GFP or Ad-dnRit; however, PD98059 completely blocked the inhibitory effects of caRit on dendritic growth (Fig. $3 G, H$ ).

Rit promotes axonal growth but inhibits dendritic growth in sympathetic neurons

Another neuronal cell type that has been widely used to study neuronal morphogenesis is the postganglionic sympathetic neuron (Glebova and Ginty, 2005). Such studies have identified fundamental differences between hippocampal and sympathetic neurons with respect to how axonal and dendritic growth is regulated. The most significant difference is that in contrast to hippocampal neurons, sympathetic neurons derived from embryonic rat superior cervical ganglia extend only a single axonal process during the first $24 \mathrm{~h}$ in culture, and when these neurons are grown in serum-free medium in the absence of non-neuronal cells, this polarity is maintained for up to 3 months in culture (Bruckenstein and Higgins, 1988). Dendritic growth in sympathetic neurons requires extrinsic cues: coculture with glial cells (Tropea et al., 1988; Lein et al., 2002) or exposure to BMPs in the presence of NGF (Lein et al., 1995) triggers these neurons to form multiple dendrites in addition to a single axon. Thus, to determine whether Rit signaling represents a divergent or convergent mechanism for regulating axonal versus dendritic growth modes in diverse neuronal cell types, we first determined whether Rit is expressed endogenously in sympathetic neurons and then analyzed the effects of manipulating Rit activation on axonal and dendritic growth in sympathetic neurons.

We have shown previously that Rit transcripts are present in purified cultures of sympathetic neurons derived from embryonic rat superior cervical ganglia (Spencer et al., 2002b). In this study, immunocytochemical analyses of primary cultures of sympathetic neurons revealed Rit immunoreactivity throughout the neuron at varying stages of morphological differentiation. At $24 \mathrm{~h}$ after plating, sympathetic neurons exhibited diffuse cytoplasmic Rit immunoreactivity throughout the neuronal cell body as well as within the shaft and growth cone of the nascent axon (Fig. 4A). Rit immunoreactivity was also evident in the filopodia extending from both the cell body and the axonal growth cone. In mature sympathetic neurons induced to elaborate a dendritic arbor by exposure to BMP7 (Lein et al., 1995), Rit antibodies 
Table 1. Effect of Rit expression on cell viability in cultured hippocampal neurons

\begin{tabular}{lll}
\hline Plasmid & Hours after infection & Percentage of viable neurons \\
\hline Ad-GFP & 24 & $83 \pm 2.5$ \\
Ad-dnRit & 24 & $88 \pm 4.2$ \\
Ad-caRit & 24 & $85 \pm 3.6$ \\
Ad-GFP & 72 & $85 \pm 2.2$ \\
Ad-dnRit & 72 & $82 \pm 3.1$ \\
Ad-caRit & 72 & $85 \pm 2.7$ \\
\hline
\end{tabular}
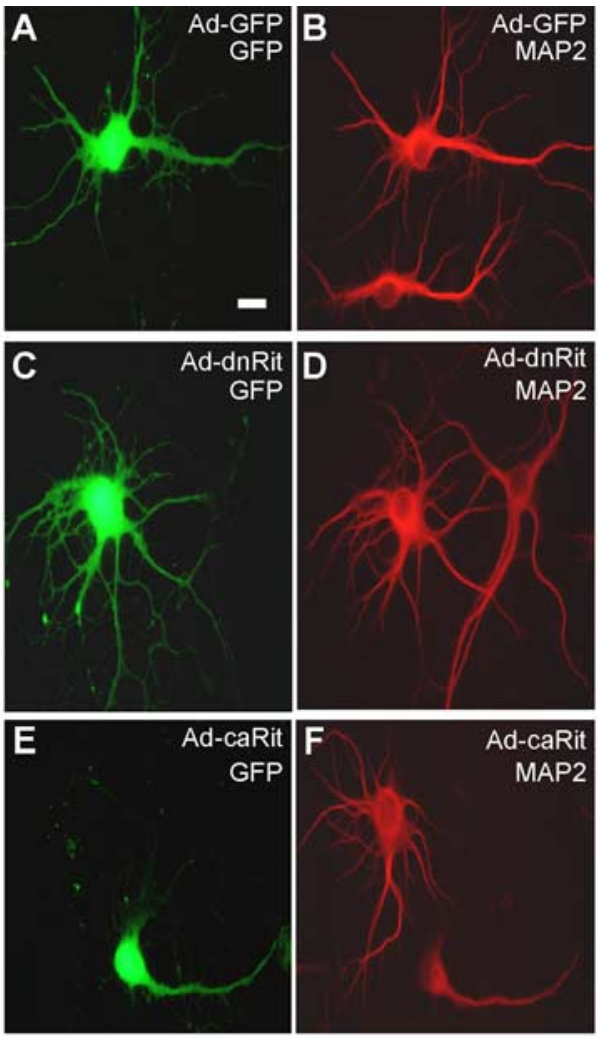

G
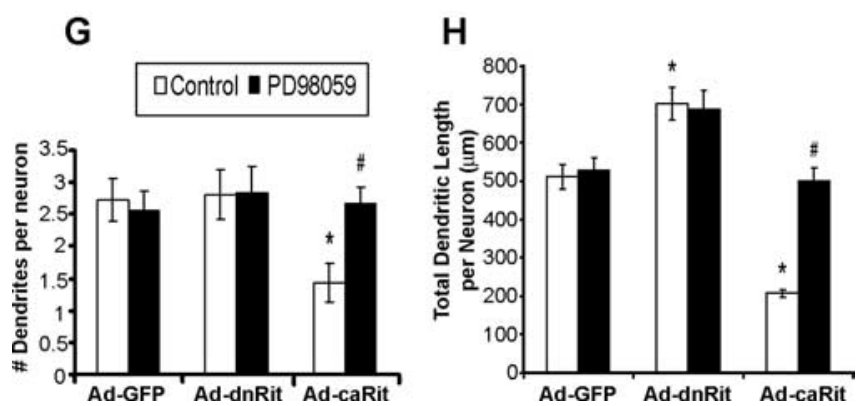

Figure 3. Rit activation inhibits dendritic growth in cultured hippocampal neurons. $\boldsymbol{A}-\boldsymbol{F}$ Hippocampal neurons were infected $16 \mathrm{~h}$ after plating with adenovirus expressing GFP alone (Ad-GFP; $\boldsymbol{A}, \boldsymbol{B}$ ) or coexpressing GFP and either dominant-negative Rit ${ }^{\mathrm{S} 35 \mathrm{~N}}$ (Ad-dnRit; $\boldsymbol{C}, \boldsymbol{D}$ ) or constitutively active Rit ${ }^{\text {Q79L }}$ (Ad-caRit; $\boldsymbol{E}, \boldsymbol{F}$ ). The MEK inhibitor PD98059 (10 $\mu \mathrm{m}$ ) was added $24 \mathrm{~h}$ after infection, and $72 \mathrm{~h}$ after infection, cultures were fixed and immunostained for both GFP (green) and the dendrite-selective antigen MAP2 (red). $\boldsymbol{G}, \boldsymbol{H}$, The number of dendrites ( $\boldsymbol{G}$ ) and total dendritic length per neuron $(\boldsymbol{H})$ were quantified in GFP + neurons. Expression of dnRit enhanced dendritic growth $(\boldsymbol{C}, \boldsymbol{D})$ relative to that observed in cultures expressing only GFP $(\boldsymbol{A}, \boldsymbol{B})$, and PD98059 had no effect on this morphogenic response $(\boldsymbol{G}, \boldsymbol{H})$. In contrast, expression of caRit significantly inhibited dendritic growth $(\boldsymbol{E}, \boldsymbol{F})$, and this effect was blocked by PD98059 $(\boldsymbol{G}, \boldsymbol{H})$. Data are expressed as the mean \pm SEM ( $n \geq 30$ neurons per experimental group). $p<$ 0.05 (ANOVA); ${ }^{*} p<0.05$ versus GFP control ( $p$ ost hoc analysis); ${ }^{\#} p<0.05$ versus Ad-caRit control ( post hoc analysis). Scale bar, $25 \mu \mathrm{m}$. stained not only the cell body and axon but also the entire extent of the dendritic arbor (Fig. 4B).

To examine the influence of Rit on axonal growth, cultured sympathetic neurons were infected with Ad-GFP, Ad-dnRit, or Ad-caRit $16 \mathrm{~h}$ after plating, and axonal growth was quantified $24 \mathrm{~h}$ after infection. Inhibition of Rit function in sympathetic neurons by dnRit expression had no effect on the number of axons extended by sympathetic neurons during the first $2 \mathrm{~d}$ in culture but did significantly decrease axonal length to approximately one-third of the mean value measured for control sympathetic neurons expressing GFP only (Fig. 5). In contrast to observations made in hippocampal neurons, activation of Rit by caRit expression altered the number of axons extended by sympathetic neurons, increasing this parameter from control levels of one axon per neuron to two to three axons per neuron. Expression of caRit also increased axonal branching, resulting in an approximate threefold increase in total axonal length per neuron (Fig. 5), similar to the effects of activated Rit on neurite outgrowth and branching in pheochromocytoma and SH-SY5Y cells (Hynds, 2003; Shi and Andres, 2005; Spencer et al, 2002b). Neither AddnRit nor Ad-caRit altered neuronal cell viability at $24 \mathrm{~h}$ after infection (Table 2), suggesting that their effects on axonal growth are not attributable to changes in cell viability.

We next determined whether Rit signaled via MEK/ERK to promote axonal growth in sympathetic neurons. Because of the lower infection efficiency in sympathetic neurons, it was not technically possible to determine whether caRit similarly increased phosphorylated ERK1/2 in sympathetic neurons. However, as in hippocampal neurons, inhibition of MEK signaling by PD98059 $(10 \mu \mathrm{M})$ or U0126 $(10 \mu \mathrm{M})$ had no effect on the morphology of neurons expressing Ad-GFP or Ad-dnRit but did block the axon-promoting effects of Ad-caRit (Fig. 5G,H).

To determine whether Rit signaling influences dendritic growth, cultured sympathetic neurons were infected with AdGFP, Ad-dnRit, or Ad-caRit 3-5 d after plating, and dendritic growth was quantified $3 \mathrm{~d}$ after infection. Previous studies of cultured sympathetic neurons have shown that $>90 \%$ of the neuronal cell population extends dendrites within $3 \mathrm{~d}$ after simultaneous exposure to both NGF and BMP7 (Lein et al., 1995). The cultures used to quantify Rit influence on dendritic growth were maintained in optimal concentrations of NGF, but BMP7 concentrations were varied from $0-10 \mathrm{ng} / \mathrm{ml}$ (suboptimal) to 50 $\mathrm{ng} / \mathrm{ml}$ (maximally effective). In the absence of BMP7, none of the adenoviral vectors (Ad-GFP, Ad-dnRit, or Ad-caRit) induced dendritic growth (data not shown). However, these adenoviral vectors did modulate dendritic growth in sympathetic neurons exposed to BMP7 immediately after infection. Sympathetic neurons infected with Ad-GFP in the presence of maximally effective concentrations of BMP7 $(50 \mathrm{ng} / \mathrm{ml})$ extended one to two tapered processes that were immunopositive for the dendrite-selective cytoskeletal protein MAP2 (Fig. 6A,B). Expression of caRit in sympathetic neurons significantly inhibited the dendritic response to these optimal BMP7 concentrations (Fig. 6E,F), as evidenced by an approximate twofold decrease in both dendritic number (Fig. 6G) and length per neuron (Fig. $6 H$ ). Expression of dnRit in neurons exposed to maximally effective concentrations of BMP7 had no effect on BMP-induced dendritic growth (Fig. $6 C, D)$ with respect to either the number or length of dendrites extended per neuron (Fig. 6G,H). One possible explanation for the lack of effect of dnRit is that levels of activated Rit in neurons exposed to maximally effective concentrations of BMPs are so low that expression of dnRit has little physiological effect. To test this possibility, sympathetic neurons infected with Ad-GFP or 

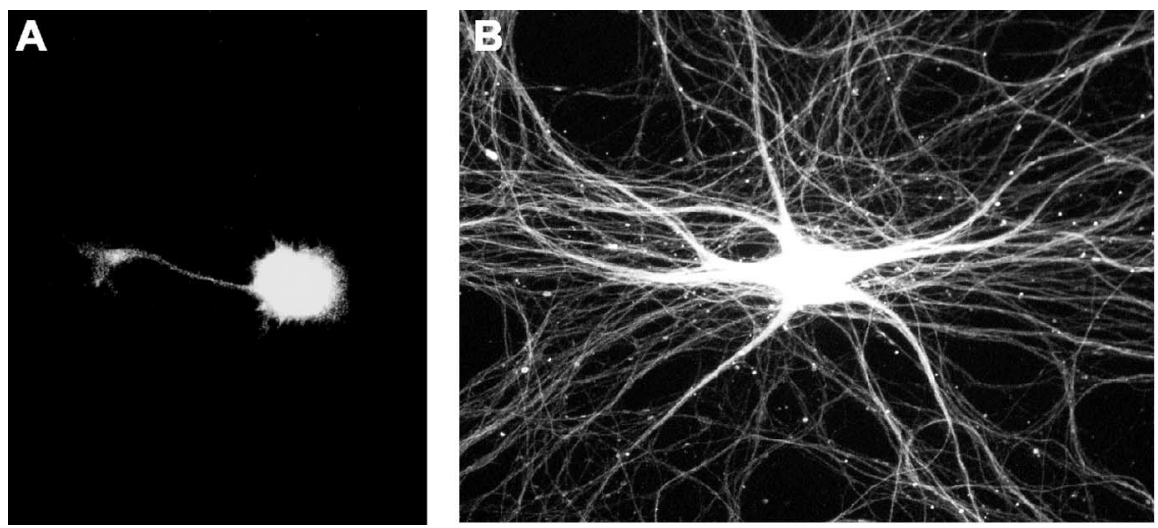

Figure 4. Subcellular localization of endogenous Rit in cultured sympathetic neurons. Fluorescence micrographs of sympathetic neurons immunostained for Rit are shown. $A$, After $24 \mathrm{~h}$ in culture, sympathetic neurons grown on poly-D-lysine typically extend a single axonal process. During these early stages of axonal growth, Rit immunoreactivity is evident in not only the cytoplasm of the neuronal somata but also throughout the axonal shaft and growth cone. Filopodia extending from the cell body and axonal growth cone are also immunopositive for Rit. $\boldsymbol{B}$, The addition of BMP7 to cultured sympathetic neurons $(50 \mathrm{ng} / \mathrm{m}$ during days 7-14 in vitro) induces the extension of multiple dendrites in addition to a single axon. In these more mature neurons, diffuse Rit immunoreactivity is present in not only the soma but also throughout the length of both axons and dendrites.

caRit decreases dendritic growth in sympathetic neurons. However, BMP7induced dendritic growth requires NGF (Lein et al., 1995), and in the exposure paradigm used in this study, sympathetic neurons were exposed to maximally effective concentrations of NGF $(100 \mathrm{ng} / \mathrm{ml})$ before and during BMP treatment. Consistent with previous studies (Shi and Andres, 2005), treatment of PC6 cells expressing 3xFlag-Rit-WT with NGF alone for $10 \mathrm{~min}, 4 \mathrm{~h}$, or $24 \mathrm{~h}$ also increased levels of Rit-GTP (Fig. 7C,D). However, in cultures pretreated with NGF for these same time periods, subsequent stimulation with BMP7 for 10 min caused a decrease in RitGTP levels (Fig. 7A,B). These changes in levels of Rit-GTP occurred in the absence of obvious changes in levels of total Rit or TrkA proteins. To confirm that cross talk between BMP7 and NGF signaling pathways resulted in the inhibition of Rit activation seen after either NGF or BMP7

Ad-dnRit were exposed to suboptimal concentrations of BMP7 ( $10 \mathrm{ng} / \mathrm{ml}$ ). A $3 \mathrm{~d}$ exposure to these suboptimal BMP concentrations elicited the extension of a single, relatively short dendrite in neurons expressing GFP only (Fig. 6I,J). Expression of dnRit potentiated the dendrite-promoting activity of this suboptimal concentration of BMP7 as evidenced by a trend toward an increasing number of dendrites per neuron (Fig. 6I) and a significant increase in total dendritic length per neuron (Fig. $6 \mathrm{~J}$ ). These effects of dnRit and caRit constructs on dendritic morphology are not likely attributable to effects on cell viability, because expression of Ad-caRit or Ad-dnRit did not alter neuronal cell viability at $72 \mathrm{~h}$ after infection relative to control cultures exposed to Ad-GFP (Table 2), and infection with Ad-GFP did not significantly alter the dendritic response of sympathetic neurons to BMP7 relative to uninfected neurons (data not shown). To investigate the role of ERK1/2 signaling in Rit effects on dendritic growth, neurons were simultaneously exposed to BMP7 and PD98059 after infection with Ad-GFP, Ad-caRit, or Ad-dnRit. In neurons infected with Ad-GFP or Ad-dnRit, PD98059 had no effect on dendritic responses; however, PD98059 completely blocked the inhibitory effects of caRit on dendritic growth (Fig. $6 G, H)$.

\section{BMP7 influences Rit activation in PC6 cells and cultured hippocampal neurons}

The observation that BMP7-induced dendritic growth in sympathetic neurons is potentiated by dnRit and significantly attenuated by caRit suggested that BMP7 signaling may trigger dendritic growth in part by increasing the ratio of inactive GDPbound Rit to active GTP-bound Rit. Rit-GTP pull-down assays were technically difficult in sympathetic neurons because of low levels of endogenous Rit protein and low infection/transfection efficiencies. Thus, to test the hypothesis that BMP7 decreases levels of activated Rit, we performed Rit-GTP pull-down assays using PC6 cells expressing 3xFlag-Rit-WT as described previously (Shi and Andres, 2005). Treatment of these PC6 cultures with maximally effective concentrations of BMP7 (30 ng/ml) for varying times ranging from $10 \mathrm{~min}$ to $24 \mathrm{~h}$ caused a significant increase in cellular levels of activated GTP-bound Rit (Fig. 7 A, B), setting up an apparent contradiction with the observation that stimulation, we repeated these studies in cultures pretreated with BMP7. As seen in Figure 7, $C$ and D, BMP7 pretreatment potently inhibited NGF-mediated Rit activation. Thus, cross talk between BMP and NGF signal transduction cascades results in a decrease in cellular Rit-GTP levels, consistent with a requirement for modulated Rit activity in BMP7-mediated dendritic growth.

BMP7 has previously been shown to enhance dendritic growth in cultured hippocampal neurons (Withers et al., 2000). Consistent with this observation, we observed that BMP7 (30 $\mathrm{ng} / \mathrm{ml}$ for $3 \mathrm{~d}$ ) significantly increased dendritic growth as evidenced by comparing the total length of the dendritic arbor in BMP7-treated $(487 \pm 72 \mu \mathrm{m})$ versus control $(224 \pm 36 \mu \mathrm{m})$ hippocampal cultures derived from the same dissection. Rit-GTP pull-down assays using hippocampal neurons expressing the 3xFlag-Rit-WT construct (60-80\% transfection efficiency using the Amaxa nucleofector) revealed that treatment of these cultures with BMP7 (30 ng/ml) for 30 min significantly decreased RitGTP levels (Fig. 7 E, F). These data are consistent with the observation that expression of caRit inhibits and dnRit potentiates dendritic growth in this neuronal cell type (Fig. 3).

\section{Discussion}

A variety of extracellular signals including trophic factors, adhesive molecules, soluble chemoattractants and chemorepellants, and neurotransmitters influence the formation and extension of axons and dendrites (Scott and Luo, 2001; Huber et al., 2003). Thus, the acquisition of axonal versus dendritic identity requires the coordinated action of multiple signaling events that culminate in axon- or dendrite-appropriate reorganization of the cytoskeleton. Signaling proteins implicated in regulating the cytoskeletal changes that underlie axonal and dendritic growth include phosphatidylinositol-3-kinase (PI3-kinase), the Akt/glycogen synthase kinase-3 $\beta$ (GSK-3 $\beta$ )/CRMP-2 [or adenomatous polyposis coli (APC)] and Par3/Par6/atypical PKC complexes, and the Ras and Rho GTPases (Arimura and Kaibuchi, 2005; Govek et al., 2005; Wiggin et al., 2005). Here, we identify Rit as the newest member of the Ras family to be involved in regulating morphogenic events in neurons. Our data demonstrate that in hippocampal and sympathetic neurons (1) endogenous Rit is expressed throughout the neuron at varying stages of morpho- 
logical differentiation, (2) overexpression of dnRit blocks axonal growth but promotes dendritic growth, and (3) overexpression of caRit enhances axonal growth but inhibits dendritic growth.

Regulation of MEK/ERK signaling is critical to the actions of many Ras-related GTPases (Reuther and Der, 2000). In agreement with this, we found that Rit activates ERK1/2 in neurons and pharmacological inhibition of MEK1 blocks both the axon-promoting and dendrite-inhibiting activity of caRit. These data are consistent with reports that MEK/ERK signaling enhances axonal growth in sympathetic (Atwal et al., 2000; Thompson et al., 2004) and hippocampal (Gerecke et al., 2004) neurons and with our previous observations that BMP7-induced dendritic growth in sympathetic neurons is potentiated by inhibition of MEK/ERK activation (Kim et al., 2004). However, our findings contrast with reports that MEK/ERK signaling mediates activitydependent increases in dendritic growth in both hippocampal (Wayman et al., 2006) and sympathetic neurons (Vaillant et al., 2002), suggesting that MEK/ ERK activation alone is not sufficient to fully explain Rit effects on axonal and dendritic growth.

The morphogenic effects of Rit are different from those of other Ras family members, supporting the possibility that factors in addition to MEK/ERK contribute to axonal and dendritic regulation by Rit. For example, Rap1B is implicated in establishing polarity in hippocampal neurons via activation of Cdc42 and the Par3/ Par6 complex; however, overexpression of caRap1B in hippocampal neurons has no effect on either the number or length of axons or dendrites (Schwamborn and Puschel, 2004). Like Rit, Ras activation causes the formation of multiple axons; in contrast to Rit, Ras regulates axon specification and promotes dendritic growth (Arendt et al., 2004; Gartner et al., 2004; Jaworski et al., 2005; Kumar et al., 2005; Yoshimura et al., 2006). The effects of $\mathrm{H}$-Ras on axon specification are mediated by the PI3-kinase/Akt/GSK-3 $\beta /$ CRMP-2 signaling pathway (Yoshimura et al., 2006). Therefore, Rit-specific signaling may be determined by differential activation of downstream effector pathways and/or the duration and subcellular distribution of activated Rit (Reuther and Der, 2000). Several downstream targets of Rit have been identified in nonneuronal cells, including B-Raf, a protein kinase that links Rit to both ERK and p38 MAP kinase pathways (Shi and Andres, 2005); RGL3, a regulator of the Ral GTP-binding proteins (Shao and Andres, 2000); AF-6, an actin-filament- and nectin-binding protein (Mandai et al., 1997); and PAR6, a component of an evolu-
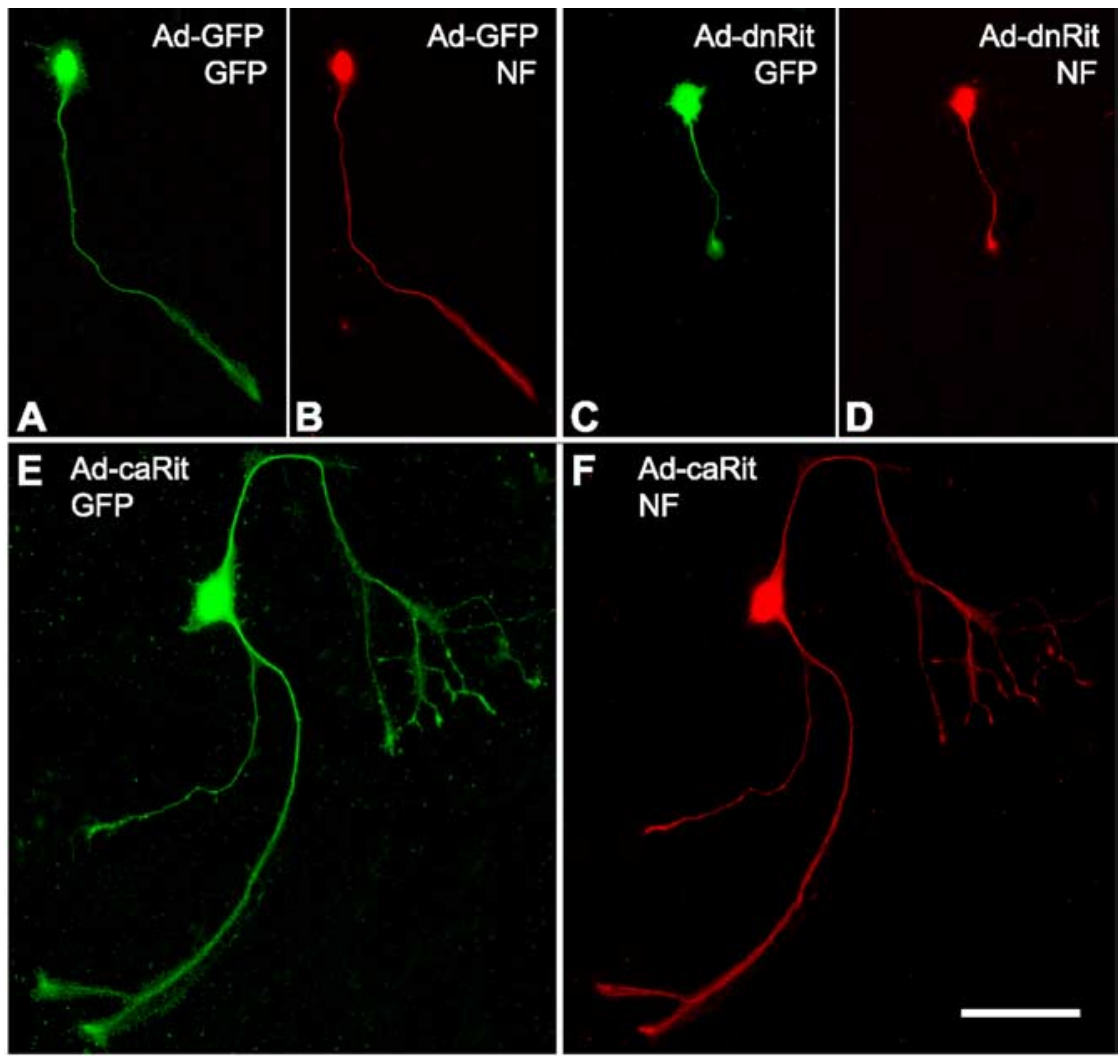

口Control aPD98059 $=0126$
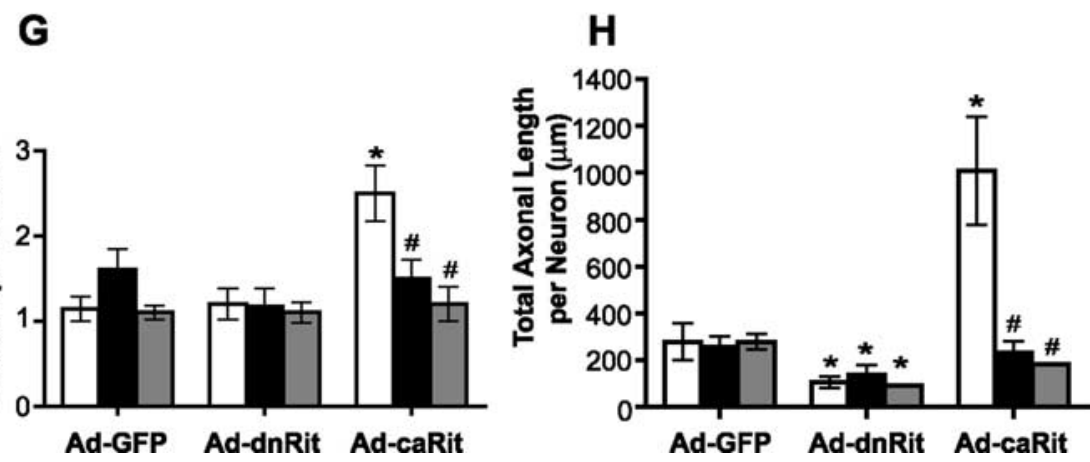

Figure 5. Rit activation promotes axonal growth in sympathetic neurons. $\boldsymbol{A}-\boldsymbol{F}$, Sympathetic neurons were infected $16 \mathrm{~h}$ after plating with adenovirus expressing GFP alone (Ad-GFP; $\boldsymbol{A}, \boldsymbol{B}$ ) or coexpressing GFP and either dominant-negative Rit ${ }^{\mathrm{S} 35 \mathrm{~N}}$ (Ad-dnRit; $\boldsymbol{C}, \boldsymbol{D})$ or constitutively active Rit ${ }^{\mathrm{Q79L}}$ (Ad-caRit; $\left.\boldsymbol{E}, \boldsymbol{F}\right)$ in the absence or presence of the MEK inhibitors PD98059 (10 $\mu \mathrm{m}$; black bars) or U0126 (10 $\mu \mathrm{m}$; gray bars). Cultures were fixed and immunostained for both GFP (green) and the phosphorylated forms of the M and $\mathrm{H}$ neurofilament (NF) subunits, an axon-selective antigen (red), $24 \mathrm{~h}$ after infection. $\boldsymbol{G}, \boldsymbol{H}$, The number of axons ( $\boldsymbol{G}$ ) and total axonal length per neuron $(\boldsymbol{H})$ were quantified in GFP + neurons. Relative to levels of axonal growth observed in control neurons expressing only GFP $(\boldsymbol{A}, \boldsymbol{B})$, expression of $\operatorname{dnRit}(\boldsymbol{C}, \boldsymbol{D})$ significantly decreased axonal length. In contrast, expression of caRit $(\boldsymbol{E}, \boldsymbol{F})$ caused somatic hypertrophy and a significant increase in axonal growth evident as an increase in the number $(\boldsymbol{G})$ and length $(\boldsymbol{H})$ of axons. The axon-promoting effects of caRit were blocked by PD98059 and by U0126 $(\mathbf{G}, \boldsymbol{H})$. Data are expressed as the mean \pm SEM ( $n \geq 30$ neurons per experimental group). $p<0.05$ (ANOVA); ${ }^{*} p<0.05$ versus GFP control ( post hoc analysis); ${ }^{\#} p<0.05$ versus caRit control ( post hoc analysis). Scale bar, $100 \mu \mathrm{m}$.

Table 2. Effect of Rit expression on cell viability in cultured sympathetic neurons

\begin{tabular}{lll}
\hline Plasmid & Hours after infection & Percentage of viable neurons \\
\hline Ad-GFP & 24 & $86 \pm 2.4$ \\
Ad-dnRit & 24 & $84 \pm 3.8$ \\
Ad-caRit & 24 & $83 \pm 4.4$ \\
Ad-GFP & 72 & $82 \pm 3.2$ \\
Ad-dnRit & 72 & $81 \pm 2.6$ \\
Ad-caRit & 72 & $84 \pm 2.8$
\end{tabular}



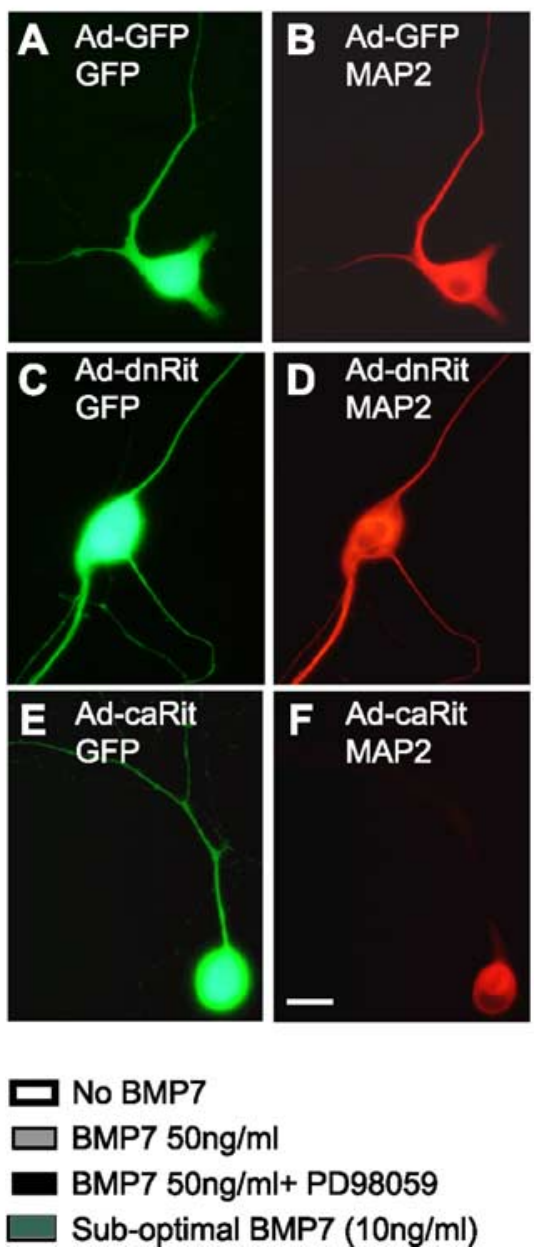

Figure 6. Rit activation inhibits BMP-induced dendritic growth in sympathetic neurons. $\boldsymbol{A}-\boldsymbol{F}$, Sympathetic neurons were infected with adenovirus expressing GFP alone (Ad-GFP; $\boldsymbol{A}, \boldsymbol{B})$ or coexpressing GFP and either dominant-negative Rit ${ }^{\mathrm{S35N}}$ (Ad$\mathrm{dnRit} ; \boldsymbol{C}, \boldsymbol{D})$ or constitutively active Rit ${ }^{\mathrm{Q79L}}$ (Ad-caRit; $\left.\boldsymbol{E}, \boldsymbol{F}\right) 3-5 \mathrm{~d}$ after plating. $\boldsymbol{H}-\boldsymbol{J}$, Within $24 \mathrm{~h}$ after infection, cultures were treated with BMP7 at either maximally effective $(50 \mathrm{ng} / \mathrm{ml} ; \boldsymbol{A}-\boldsymbol{H})$ or suboptimal $(10 \mathrm{ng} / \mathrm{ml} \boldsymbol{I}, \boldsymbol{J})$ concentrations in the absence or presence of the MEK inhibitor PD98059 (10 $\mu \mathrm{M}) . \mathbf{G}, \boldsymbol{H}$, After a 3 d exposure to BMP7, cultures were fixed and immunostained for GFP (green) and the dendrite-selective antigen MAP2 (red), and the number of dendrites $(\boldsymbol{G})$ and total dendritic length per neuron $(\boldsymbol{H})$ was quantified in GFP + neurons. Relative to levels of dendritic growth observed in control neurons expressing only GFP $(\boldsymbol{A}, \boldsymbol{B})$, expression of $\operatorname{dnRit}(\boldsymbol{C}, \boldsymbol{D})$ caused little or no effect on dendritic growth induced by maximally effective concentrations of BMP7 ( $\boldsymbol{G}$, $\boldsymbol{H})$. However, dnRit did potentiate dendritic growth in the presence of suboptimal concentrations of BMP7 $(\boldsymbol{I}, \boldsymbol{J})$. Expression of caRit significantly inhibited dendritic growth induced by maximally effective concentrations $(\boldsymbol{E}, \boldsymbol{F})$, and this inhibitory effect was blocked by PD98059 (G, H). Data are expressed as the mean \pm SEM ( $n \geq 30$ neurons per experimental group). $p<0.05$ (ANOVA); ${ }^{*} p<0.05$ versus BMP7-treated neurons expressing GFP alone ( post hoc analysis); ${ }^{\#} p<0.05$ versus BMP7-treated neurons expressing caRit ( post hoc analysis). Scale bar, $50 \mu \mathrm{m}$.

tionarily conserved complex involved in determining polarity in many cell types (Fukata et al., 2003) that is also involved in axonal specification (Shi et al., 2003). Whether these effectors are required for Rit-mediated control of axonal and dendritic growth remains to be determined.

Additional downstream signaling molecules that mediate morphogenic effects of Rit are likely to include CREB (cAMP response element-binding protein), a target of MEK/ERK signaling known to influence neuronal morphogenesis (Lonze and Ginty, 2002) and, ultimately, Rho GTPases, which regulate actin and microtubule dynamics. The Rho GTPases, including the well characterized Rac, Cdc42, and RhoA proteins, have been implicated in regulating axonal and dendritic growth responses to a variety of signals (Threadgill et al., 1997; Hall, 1998; Luo, 2000; Govek et al., 2005). Cdc42 and Racl activity is most often associ- ated with neurite extension, whereas RhoA is implicated in neurite retraction (for review, see Govek et al., 2005). The activity of Rho GTPases is controlled by their interaction with specific guanine nucleotide exchange factors (GEFs) and GTPase-activating proteins (GAPs). For example, association of the Rac-specific GEFs Tiam-1/STEF with the Par3/Par6/ $\alpha \mathrm{PKC}$ complex results in localized and coupled activation of both $\mathrm{Cdc} 42$ and Rac1 GTPases to promote axon specification and elongation (Shi et al., 2003; Nishimura et al., 2005), whereas activation of the Trio and Kalirin Rho-family GEFs activates RhoG to promote axon initiation (Bateman and Van Vactor, 2001; Estrach et al., 2002; May et al., 2002). Kalirin also plays a prominent role in the maintenance of dendrites and dendritic spines in hippocampal pyramidal neurons (Ma et al., 2003), suggesting that spatial and temporal regulation of Rho-family GTPases and cross talk between Rho GTPases is crucial to axon-dendrite differentiation. Future studies are needed to identify relevant interactions between Rit, Rho GTPases, GEFs, and GAPs.

Also critical to understanding how Rit signaling controls neuronal morphogenesis is identifying upstream signaling events that modulate Rit activation. We previously demonstrated that NGF activates Rit (Shi and Andres, 2005), and in this study, we found that BMP7 regulates Rit activity. The kinetics of Rit regulation are consistent with a BMP receptor-initiated signaling pathway directly modulating Rit activation as we reported previously for NGF activation of Rit via TrkA signaling (Spencer et al., 2002b). Although a great deal is known about BMP signaling via SMAD (mothers against decapentaplegic)mediated gene regulation, much less is understood about nontranscriptional signaling by BMPs. BMPs regulate a variety of MAP kinase signaling cascades (Massague and Gomis, 2006), and recent studies describe SMAD-independent links between BMP receptors and Lim kinase-1 (Lee-Hoeflich et al., 2004) and glutamate (Shen et al., 2004) receptors. To our knowledge, our evidence suggesting Rit as a novel downstream target of BMP signaling is the first report linking BMP signaling to activation of a Rasrelated GTPase and may provide a novel mechanism for coupling $\mathrm{BMP}$ receptors to MAP kinase signaling pathways.

In hippocampal neurons, BMP7 decreased levels of GTPbound Rit, consistent with enhanced dendritic growth in response to BMP7 or dnRit. In contrast, in PC6 cells, BMP7 increased levels of GTP-bound Rit. Because PC6 cells are widely used as a model of sympathetic neurons, this was unexpected because the present study suggests that Rit activation inhibits BMP7-mediated dendritic growth in sympathetic neurons. However, when BMP7 was added to PC6 cells pretreated with NGF, or 
conversely, NGF added following pretreatment with BMP7, levels of activated Rit were decreased. The molecular mechanism(s) mediating this NGF/BMP cross talk in the regulation of Rit activation is presently unclear, but the available data provide important clues. The rapid onset of NGF/BMP-mediated inhibition of Rit activation with no obvious change in total Rit protein expression suggests that neither transcriptional regulation nor protein turnover are involved. TrkA protein levels also are unchanged during these exposures, suggesting that decreased RitGTP levels are not attributable to decreased NGF signaling. Two potential regulatory models include transient inactivation of a Rit GEF or activation of a Rit GAP resulting in downregulated Rit signaling. There are examples of dynamic activation/inactivation of these important regulatory proteins via protein phosphorylation or translocation between subcellular compartments (Rossman et al., 2005). To date, no GEF/GAP regulatory proteins have been identified for Rit; however, a variety of Ras family GEF and GAP proteins are expressed in neurons. Identifying Rit GEFs and GAPs and determining their role(s) in NGF-, BMP7-, and NGF/BMP7dependent Rit regulation are important issues to be addressed.

Although the molecular mechanisms underlying the paradoxical effects of combined NGF and BMP signaling on Rit activation are not known at this time, the observation is consistent with previous reports that in sympathetic neurons, ERK1/2 signaling negatively impacts BMP7-induced dendritic growth (Kim et al., 2004) and that BMP7-induced dendritic growth requires NGF (Lein et al., 1995). Moreover, it has interesting impli-

cations regarding the role of NGF signaling in dendritic growth in sympathetic neurons. Specifically, our data suggest that in addition to maintaining the trophic state of the neuron, which is critical for dendritic growth in sympathetic neurons (Lein et al., 1995), NGF signaling actively contributes to unique signaling pathways that trigger dendritic growth.

Could opposing effects of Rit on axonal and dendritic growth represent a mechanism for regulating changes in the intrinsic growth state of neurons? Our data suggest that the relative ratio of GTP- versus GDP-bound Rit may influence the intrinsic growth state: when this ratio is $>1$, neurons are in axonal growth mode; when this ratio is $<1$, the dendritic growth mode is favored. Retinal ganglia cells represent a relatively unique model in which the morphogenic response to neurotrophins stably switches from an axonal to dendritic growth mode during development (Goldberg et al., 2002), and it would be of interest to determine whether this switch corresponds to global changes in the activational status of Rit. However, most neurons, including hippocampal and sympathetic neurons, continue to elongate axons while dendrites are elaborating, thus it seems likely that Rit signaling differen-

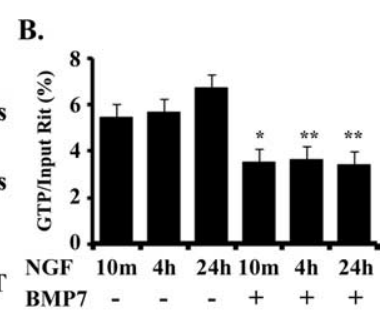

D.
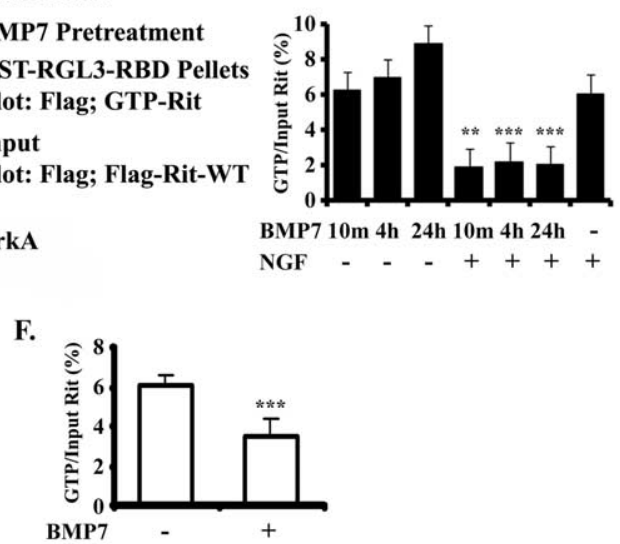

Figure 7. BMP7 treatment decreases GTP loading of Rit in PC6 cells pretreated with NGF and in hippocampal neurons. GST pull-down assays using GST-RGL3-RBD agarose were performed using PC6 cells $(\boldsymbol{A}-\boldsymbol{D})$ or hippocampal neurons $(\boldsymbol{E}, \boldsymbol{F})$ expressing 3xFlag-Rit-WT. The levels of GTP-bound Rit were determined by immunoblot analysis using anti-Flag mAb. PC6 cells were serum

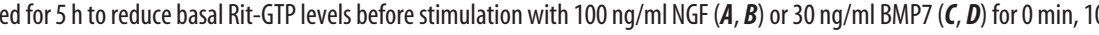
activation, PC6 cells expressing 3xFlag-Rit-WT were either pretreated with NGF (100 ng/ml) for $10 \mathrm{~min}, 4 \mathrm{~h}$, or $24 \mathrm{~h}$ before $(100 \mathrm{ng} / \mathrm{ml})$ for $10 \mathrm{~min}$ (lanes 5-7) (C, D). For BMP7 treatment $(\boldsymbol{A})$, either $200 \mu \mathrm{g}$ (High) or $100 \mu \mathrm{g}$ (Low) of total cell lysate were examined to determine Rit-GTP levels. Cross talk between BMP7- and NGF-signaling pathways appears to inhibit Rit activation,

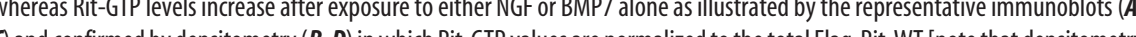
of data in $\boldsymbol{A}$ was performed using data obtained with the lower amounts $(100 \mu \mathrm{g})$ of total cell lysate]. $\boldsymbol{E}, \boldsymbol{F}$, Hippocampal neurons were transfected with 3xFlag-Rit-WT at the time of plating, maintained in serum-free Neurobasal medium supplemented with B27 for $72 \mathrm{~h}$, and either left untreated or treated with $30 \mathrm{ng} / \mathrm{ml} \mathrm{BMP7} \mathrm{for} 30 \mathrm{~min}$. BMP7 inhibits Rit activation as illustrated in a resentative blot $(\boldsymbol{E})$ and confirmed by densitometry $(\boldsymbol{F})$ in which values obtained for Rit-GTP are normalized to total Flag experiments performed using two different sets of cultures. Densitometry data are expressed as mean + SEM ( $n=2$ per experimental condition). ${ }^{*} p<0.05 ;{ }^{* *} p<0.005 ;{ }^{* * *} p<0.001$ ( $t$ test).

tially controls axonal versus dendritic growth via local changes in Rit activity. Precedence for this type of mechanism comes from observations that local changes in cGMP levels convert the response of individual growth cones to semaphorins from repulsion to attraction (Song et al., 1998) and underlie the differential effect of semaphorins on axonal versus dendritic growth cones (Polleux et al., 2000).

In summary, our data identify Rit as a novel target in cross talk between NGF and BMP signaling pathways and suggest a role for Rit in regulating axonal and dendritic growth via activation of the ERK1/2 signaling pathway. Modulating the activational status of Rit may function as a molecular switch to regulate axonal versus dendritic growth responses in response to environmental cues.

\section{References}

Arendt T, Gartner U, Seeger G, Barmashenko G, Palm K, Mittmann T, Yan L, Hummeke M, Behrbohm J, Bruckner MK, Holzer M, Wahle P, Heumann R (2004) Neuronal activation of Ras regulates synaptic connectivity. Eur J Neurosci 19:2953-2966.

Arimura N, Kaibuchi K (2005) Key regulators in neuronal polarity. Neuron 48:881-884. 
Atwal JK, Massie B, Miller FD, Kaplan DR (2000) The TrkB-Shc site signals neuronal survival and local axon growth via MEK and P13-kinase. Neuron 27:265-277.

Bartlett WP, Banker GA (1984) An electron microscopic study of the development of axons and dendrites by hippocampal neurons in culture. I. Cells which develop without intercellular contacts. J Neurosci 4:1944-1953.

Bateman J, Van Vactor D (2001) The Trio family of guanine-nucleotideexchange factors: regulators of axon guidance. J Cell Sci 114:1973-1980.

Bruckenstein DA, Higgins D (1988) Morphological differentiation of embryonic rat sympathetic neurons in tissue culture. I. Conditions under which neurons form axons but not dendrites. Dev Biol 128:324-336.

DeFelipe J, Jones EG (1988) Cajal on the cerebral cortex: an annotated translation of the complete writings. New York: Oxford UP.

Dotti CG, Banker GA (1987) Experimentally induced alteration in the polarity of developing neurons. Nature 330:254-256.

Estrach S, Schmidt S, Diriong S, Penna A, Blangy A, Fort P, Debant A (2002) The human Rho-GEF trio and its target GTPase RhoG are involved in the NGF pathway, leading to neurite outgrowth. Curr Biol 12:307-312.

Fink CC, Bayer KU, Myers JW, Ferrell Jr JE, Schulman H, Meyer T (2003) Selective regulation of neurite extension and synapse formation by the beta but not the alpha isoform of CaMKII. Neuron 39:283-297.

Fukata M, Nakagawa M, Kaibuchi K (2003) Roles of Rho-family GTPases in cell polarisation and directional migration. Curr Opin Cell Biol 15:590-597.

Gartner U, Alpar A, Seeger G, Heumann R, Arendt T (2004) Enhanced Ras activity in pyramidal neurons induces cellular hypertrophy and changes in afferent and intrinsic connectivity in synRas mice. Int J Dev Neurosci 22:165-173.

Gerecke KM, Wyss JM, Carroll SL (2004) Neuregulin-1beta induces neurite extension and arborization in cultured hippocampal neurons. Mol Cell Neurosci 27:379-393.

Glebova NO, Ginty DD (2005) Growth and survival signals controlling sympathetic nervous system development. Annu Rev Neurosci 28:191-222.

Goldberg JL (2004) Intrinsic neuronal regulation of axon and dendrite growth. Curr Opin Neurobiol 14:551-557.

Goldberg JL, Klassen MP, Hua Y, Barres BA (2002) Amacrine-signaled loss of intrinsic axon growth ability by retinal ganglion cells. Science 296:1860-1864.

Goslin K, Banker G (1989) Experimental observations on the development of polarity by hippocampal neurons in culture. J Cell Biol 108:1507-1516.

Goslin K, Asmussen H, Banker G (1998) Rat hippocampal neurons in lowdensity culture. In: Culturing nerve cells, Ed 2 (Banker G, Goslin K, eds), pp 339-370. Cambridge, MA: MIT.

Govek EE, Newey SE, Van Aelst L (2005) The role of the Rho GTPases in neuronal development. Genes Dev 19:1-49.

Hall A (1998) Rho GTPases and the actin cytoskeleton. Science 279:509-514.

Higgins D, Lein PJ, Osterhout DJ, Johnson MI (1991) Tissue culture of mammalian autonomic neurons. In: Culturing nerve cells, Ed 1 (Banker G, Goslin K, eds), pp 177-206. Cambridge, MA: MIT.

Higgins D, Burack M, Lein P, Banker G (1997) Mechanisms of neuronal polarity. Curr Opin Neurobiol 7:599-604.

Hoshino M, Yoshimori T, Nakamura S (2005) Small GTPase proteins Rin and Rit Bind to PAR6 GTP-dependently and regulate cell transformation. J Biol Chem 280:22868-22874.

Howard AS, Fitzpatrick R, Pessah I, Kostyniak P, Lein PJ (2003) Polychlorinated biphenyls induce caspase-dependent cell death in cultured embryonic rat hippocampal but not cortical neurons via activation of the ryanodine receptor. Toxicol Appl Pharmacol 190:72-86.

Howard AS, Bucelli R, Jett DA, Bruun D, Yang D, Lein PJ (2005) Chlorpyrifos exerts opposing effects on axonal and dendritic growth in primary neuronal cultures. Toxicol Appl Pharmacol 207:112-124.

Huber AB, Kolodkin AL, Ginty DD, Cloutier JF (2003) Signaling at the growth cone: ligand-receptor complexes and the control of axon growth and guidance. Annu Rev Neurosci 26:509-563.

Hynds DL, Spencer ML, Andres DA, Snow DM (2003) Rit promotes MEKindependent neurite branching in human neuroblastoma cells. J Cell Sci 116:1925-1935.

Jaworski J, Spangler S, Seeburg DP, Hoogenraad CC, Sheng M (2005) Con- trol of dendritic arborization by the phosphoinositide-3'-kinase-Aktmammalian target of rapamycin pathway. J Neurosci 25:11300-11312.

Kim IJ, Beck HN, Lein PJ, Higgins D (2002) Interferon $\gamma$ induces retrograde dendritic retraction and inhibits synapse formation. J Neurosci 22:4530-4539.

Kim IJ, Drahushuk KM, Kim WY, Gonsiorek EA, Lein P, Andres DA, Higgins D (2004) Extracellular signal-regulated kinases regulate dendritic growth in rat sympathetic neurons. J Neurosci 24:3304-3312.

Kumar V, Zhang MX, Swank MW, Kunz J, Wu GY (2005) Regulation of dendritic morphogenesis by Ras-PI3K-Akt-mTOR and Ras-MAPK signaling pathways. J Neurosci 25:11288-11299.

Lee CH, Della NG, Chew CE, Zack DJ (1996) Rin, a neuron-specific and calmodulin-binding small G-protein, and Rit define a novel subfamily of ras proteins. J Neurosci 16:6784-6794.

Lee-Hoeflich ST, Causing CG, Podkowa M, Zhao X, Wrana JL, Attisano L (2004) Activation of LIMK1 by binding to the BMP receptor, BMPRII, regulates BMP-dependent dendritogenesis. EMBO J 23:4792-4801.

Lein P, Johnson M, Guo X, Rueger D, Higgins D (1995) Osteogenic protein-1 induces dendritic growth in rat sympathetic neurons. Neuron 15:597-605.

Lein PJ, Banker GA, Higgins D (1992) Laminin selectively enhances axonal growth and accelerates the development of polarity by hippocampal neurons in culture. Brain Res Dev Brain Res 69:191-197.

Lein PJ, Beck HN, Chandrasekaran V, Gallagher PJ, Chen HL, Lin Y, Guo X, Kaplan PL, Tiedge H, Higgins D (2002) Glia induce dendritic growth in cultured sympathetic neurons by modulating the balance between bone morphogenetic proteins (BMPs) and BMP antagonists. J Neurosci 22:10377-10387.

Lonze BE, Ginty DD (2002) Function and regulation of CREB family transcription factors in the nervous system. Neuron 35:605-623.

Luo L (2000) Rho GTPases in neuronal morphogenesis. Nat Rev Neurosci 1:173-180.

Ma XM, Huang J, Wang Y, Eipper BA, Mains RE (2003) Kalirin, a multifunctional Rho guanine nucleotide exchange factor, is necessary for maintenance of hippocampal pyramidal neuron dendrites and dendritic spines. J Neurosci 23:10593-10603.

Mandai K, Nakanishi H, Satoh A, Obaishi H, Wada M, Nishioka H, Itoh M, Mizoguchi A, Aoki T, Fujimoto T, Matsuda Y, Tsukita S, Takai Y (1997) Afadin: a novel actin filament-binding protein with one PDZ domain localized at cadherin-based cell-to-cell adherens junction. J Cell Biol 139:517-528

Massague J, Gomis RR (2006) The logic of TGFbeta signaling. FEBS Lett 580:2811-2820

May V, Schiller MR, Eipper BA, Mains RE (2002) Kalirin Dbl-homology guanine nucleotide exchange factor 1 domain initiates new axon outgrowths via RhoG-mediated mechanisms. J Neurosci 22:6980-6990.

McAllister AK (2000) Cellular and molecular mechanisms of dendrite growth. Cereb Cortex 10:963-973.

Nishimura T, Yamaguchi T, Kato K, Yoshizawa M, Nabeshima Y, Ohno S, Hoshino M, Kaibuchi K (2005) PAR-6-PAR-3 mediates Cdc42-induced Rac activation through the Rac GEFs STEF/Tiam1. Nat Cell Biol 7:270-277.

Polleux F, Morrow T, Ghosh A (2000) Semaphorin 3A is a chemoattractant for cortical apical dendrites. Nature 404:567-573.

Prochiantz A (1995) Neuronal polarity: giving neurons heads and tails. Neuron 15:743-746.

Redmond L, Kashani AH, Ghosh A (2002) Calcium regulation of dendritic growth via CaM kinase IV and CREB-mediated transcription. Neuron 34:999-1010.

Reuther GW, Der CJ (2000) The Ras branch of small GTPases: Ras family members don't fall far from the tree. Curr Opin Cell Biol 12:157-165.

Rossman KL, Der CJ, Sondek J (2005) GEF means go: turning on RHO GTPases with guanine nucleotide-exchange factors. Nat Rev Mol Cell Biol 6:167-180.

Schwamborn JC, Puschel AW (2004) The sequential activity of the GTPases Rap1B and Cdc42 determines neuronal polarity. Nat Neurosci 7:923-929.

Scott EK, Luo L (2001) How do dendrites take their shape? Nat Neurosci 4:359-365

Shao H, Andres DA (2000) A novel RalGEF-like protein, RGL3, as a candidate effector for Rit and Ras. J Biol Chem 275:26914-26924.

Shao H, Kadono-Okuda K, Finlin BS, Andres DA (1999) Biochemical char- 
acterization of the Ras-related GTPases Rit and Rin. Arch Biochem Biophys 371:207-219.

Shen W, Finnegan S, Lein P, Sullivan S, Slaughter M, Higgins D (2004) Bone morphogenetic proteins regulate ionotropic glutamate receptors in human retina. Eur J Neurosci 20:2031-2037.

Shi GX, Andres DA (2005) Rit contributes to nerve growth factor-induced neuronal differentiation via activation of B-Raf-extracellular signalregulated kinase and p38 mitogen-activated protein kinase cascades. Mol Cell Biol 25:830-846.

Shi SH, Jan LY, Jan YN (2003) Hippocampal neuronal polarity specified by spatially localized mPar3/mPar6 and PI 3-kinase activity. Cell 112:63-75.

Song H, Ming G, He Z, Lehmann M, McKerracher L, Tessier-Lavigne M, Poo M (1998) Conversion of neuronal growth cone responses from repulsion to attraction by cyclic nucleotides. Science 281:1515-1518.

Spencer ML, Shao H, Tucker HM, Andres DA (2002a) Nerve growth factordependent activation of the small GTPase Rin. J Biol Chem 277:17605-17615.

Spencer ML, Shao H, Andres DA (2002b) Induction of neurite extension and survival in pheochromocytoma cells by the Rit GTPase. J Biol Chem 277:20160-20168.

Thompson J, Dolcet X, Hilton M, Tolcos M, Davies AM (2004) HGF promotes survival and growth of maturing sympathetic neurons by PI-3 kinase- and MAP kinase-dependent mechanisms. Mol Cell Neurosci 27:441-452.

Threadgill R, Bobb K, Ghosh A (1997) Regulation of dendritic growth and remodeling by Rho, Rac, and Cdc42. Neuron 19:625-634.
Tropea M, Johnson MI, Higgins D (1988) Glial cells promote dendritic development in rat sympathetic neurons in vitro. Glia 1:380-392.

Vaillant AR, Zanassi P, Walsh GS, Aumont A, Alonso A, Miller FD (2002) Signaling mechanisms underlying reversible, activity-dependent dendrite formation. Neuron 34:985-998.

Vaughan PJ, Pike CJ, Cotman CW, Cunningham DD (1995) Thrombin receptor activation protects neurons and astrocytes from cell death produced by environmental insults. J Neurosci 15:5389-5401.

Wayman GA, Kaech S, Grant WF, Davare M, Impey S, Tokumitsu H, Nozaki N, Banker G, Soderling TR (2004) Regulation of axonal extension and growth cone motility by calmodulin-dependent protein kinase I. J Neurosci 24:3786-3794.

Wayman GA, Impey S, Marks D, Saneyoshi T, Grant WF, Derkach V, Soderling TR (2006) Activity-dependent dendritic arborization mediated by CaM-kinase I activation and enhanced CREB-dependent transcription of Wnt-2. Neuron 50:897-909.

Wes PD, Yu M, Montell C (1996) RIC, a calmodulin-binding Ras-like GTPase. EMBO J 15:5839-5848.

Wiggin GR, Fawcett JP, Pawson T (2005) Polarity proteins in axon specification and synaptogenesis. Dev Cell 8:803-816.

Withers GS, Higgins D, Charette M, Banker G (2000) Bone morphogenetic protein-7 enhances dendritic growth and receptivity to innervation in cultured hippocampal neurons. Eur J Neurosci 12:106-116.

Yoshimura T, Arimura N, Kawano Y, Kawabata S, Wang S, Kaibuchi K (2006) Ras regulates neuronal polarity via the PI3-kinase/Akt/GSK3beta/CRMP-2 pathway. Biochem Biophys Res Commun 340:62-68. 